\title{
Design and fabrication of CMOS optical modulator
}

\author{
Ching-Liang Dai ${ }^{\mathrm{a},}$, Hung-Lin Chen ${ }^{\mathrm{b}}$, Liang-Bin Yu ${ }^{\mathrm{b}}$, Chun-Hui Lin ${ }^{\mathrm{b}}$, Pei-Zen Chang ${ }^{\mathrm{b}}$ \\ ${ }^{a}$ Department of Mechanical Engineering, Oriental Institute of Technology, No. 58, Sze-Chuan Road, Sec. 2, Pan-Chiao, Taipei Hsien 22064, Taiwan, ROC \\ ${ }^{\mathrm{b}}$ Institute of Applied Mechanics, National Taiwan University, Taipei 107, Taiwan, ROC
}

Accepted 11 September 2001

\begin{abstract}
This study describes a micromachined optical modulator with electrostatic actuation, fabricated by the conventional CMOS process. The optical modulator is operated by the interaction of fixed stationary gratings and movable sliding gratings. The period of the gratings is determined by the slide of the movable part, allowing different diffraction patterns of reflected light. In addition, $100 \%$ modulation in the first order can act as an optical switch. All procedures following the CMOS process require a simple post-process. Maskless dry etching was the only requirement of the post-process to obtain a high-aspect-ratio microstructure, and only a low voltage of $20 \mathrm{~V}$ was necessary to drive the actuator of the optical modulator. The micromachined optical modulator proposed herein is smaller and weighs less than commercially available acoustic optical modulators. (C) 2001 Published by Elsevier Science B.V.
\end{abstract}

Keywords: Micromachine; Modulator; Gratings; CMOS

\section{Introduction}

Advances in semiconductor fabrication technology have inspired a new generation of micro-electro-mechanical systems (MEMS) [1,2] designed by using microfabrication equipment and processing methods. Optical devices are one among the most important applications of MEMS because miniaturization of the system is useful for small light spots, in supporting quick responses, low power consumption and specific work that can not be done with traditional optical elements. Accordingly, optical switches and modulators have also received considerable interest.

A modulator situated between the source and the observation point influences physical variables. An optical system includes variables such as amplitude, frequency, phase, polarization or intensity. Therefore, the optical modulator is useful in industrial applications. For instance, an acoustic optical modulator can be used as an optical switch, a scanner, a filter or an isolator [3]. Micromachined optical modulators have also attracted much attention [4]. The light is modulated by three types of mechanical deformation: vertical [5], horizontal [6] and rotatory [7-9]. The optical modulator proposed herein is of the horizontal type that operates in-plane. The stationary part in the new design is strongly fixed to the anchor, and

\footnotetext{
* Corresponding author. Tel.: +886-2-29610145x331; fax: +886-2-29543648.

E-mail addresses: fd032@ica.oit.edu.tw, q4509@ms42.hiner.net (C.-L. Dai).
}

the precision etching control is unnecessary, in contrast to the type of micromachined optical modulator reported in Transducers'99 [10]. The configuration of the modulator and a simulation of the optical modulation are described.

\section{Device design}

\subsection{Operational principles}

A periodic shape designed to variably transmit or reflect light is called a diffraction grating. Patterns differ according to the wavelength, incident angle, depth and period of the grating. The concept can be described by the grating Eq. (1), which is established by considering the path difference for the optical waves. For light incident at an angle, $\theta_{i}$, and diffracted at angle, $\theta_{m}$, the relation can be expressed as

$\sin \theta_{m}-\sin \theta_{i}=\frac{m \lambda}{a}, \quad m=0, \pm 1, \pm 2, \ldots$

where $m$ is the order number, $\lambda$ the wavelength of the incident light, and $a$ the period of the grating.

The intensity of different orders is also determined by Eq. (1). Therefore, the micromachined optical modulator proposed here simply alters the period of the gratings to modulate the incident light. The modulator with actuation as illustrated in Fig. 1 moves the position of the gratings. Consequently, the optical intensity is redistributed among the orders as the period of the grating changes. Furthermore, 


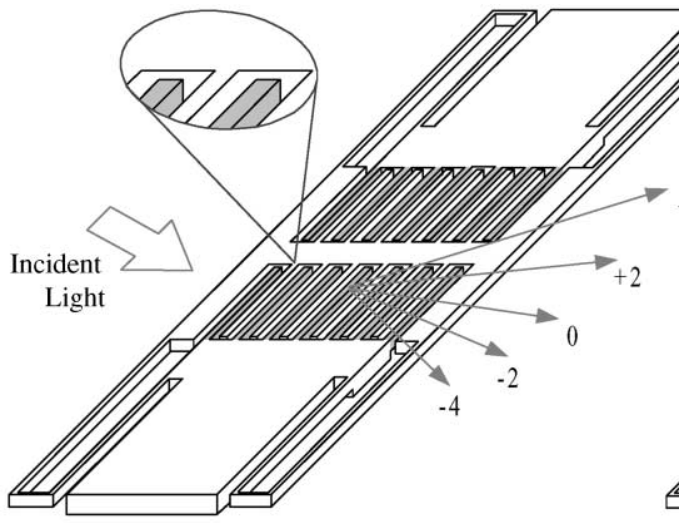

(a)

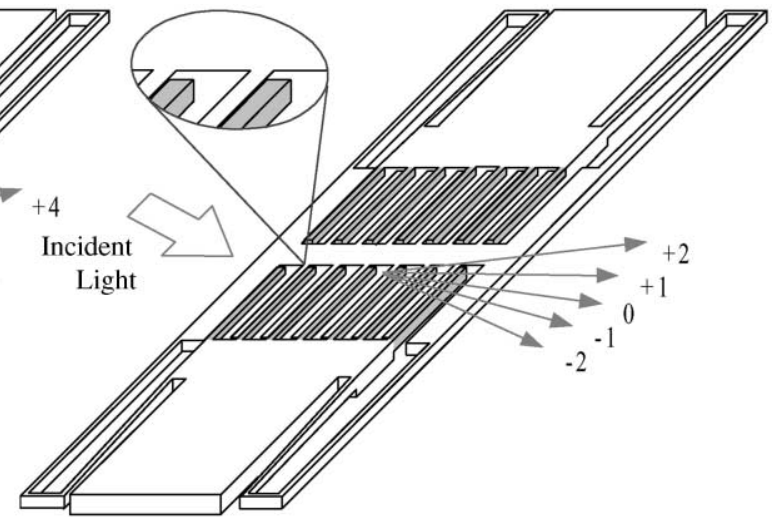

(b)

Fig. 1. Micromachined optical modulator (a) without; (b)with actuation. The intensity of reflected light redistributes to different order with mechanical driving.

in some particular orders, light is fully turned on or off and thus, the device can also serve as an optical switch.

\subsection{Requirements and characteristics}

The micromachined optical modulator must satisfy several performance and structural requirements. First, it should efficiently modulate the optical intensity, according to the shape of the gratings. Computer software is used to assess the intensity performance and the design is, thus, modified to obtain a higher efficiency.

The second requirement is the stiffness. The structure must have a high $z$-directional stiffness to avert unwanted vibrations, and low in-plane stiffness to require a lower driving voltage. This work adopts a laminated high-aspect-ratio microstructure consisting of metal and oxide layers involving the CMOS process to satisfy these requirements [11].

Easy fabrication is also important. Considerable time can be saved in developing related technologies since the CMOS process is already a mature technology and foundry services for it already exist. The post-process proposed herein requires only maskless etching to release the microstructure, facilitating actualization of the full device.

Integration is further required. The optical modulator can be integrated with operating circuits in monolithic chips since the proposed design corresponds to the CMOS process. This feature not only reduces to induce the noise of interface, but also simply constructs the final package of chips without the need for wire bonding.

The final requirement is low cost. As mentioned above, a relatively short development time effectively responds to fluctuating market demands. A monolithic chip to achieve a high performance, a batch processing and a lower manufacturing cost.

\subsection{Diffraction efficiency}

The micromachined optical modulator, consisting of sliding and stationary gratings, allows the period of the gratings to be changed by electrostatic actuation to modulate the incident light. Different diffraction patterns of reflected light are observed for the same incident light. The diffraction pattern and efficiency can be theoretically determined using the approximate physical model of the idealized pitchvarying grating. Suppose, the grating vector is in the $x$ direction, and the optical axis is in the $z$-direction, then some functions and operators can be defined [12]:

$\operatorname{comb}(s x)=\sum_{n=-\infty}^{+\infty} \delta(s x-n)=\frac{1}{s} \sum_{n=-\infty}^{+\infty} \delta\left(x-\frac{n}{s}\right)$

where $n$ is an integer, $s$ a scale constant, and $\delta(x)$ the delta function.

$\operatorname{rect}(x)= \begin{cases}1, & \text { as }-\frac{1}{2} \leq x \leq \frac{1}{2} \\ 0, & \text { otherwise }\end{cases}$

$\operatorname{sinc}(x)=\frac{\sin (\pi x)}{\pi x}$

Consider the diffracted field distribution near the grating. The complete grating consists of a stationary and a sliding grating. If $a, b$ and $d$ are the physical parameters of the pitchvarying grating shown in Fig. 2, then the field distribution on a specific plane can be simply described by,

$$
\begin{aligned}
W(x)= & \frac{1}{a} \operatorname{comb}\left(\frac{x}{a}\right) * \operatorname{rect}\left(\frac{x}{b}\right) \\
& +\frac{1}{a} \operatorname{comb}\left(\frac{x+(a / 2)+d}{a}\right) * \operatorname{rect}\left(\frac{x}{b}\right)
\end{aligned}
$$

where the asterisk $(*)$ stands for "convolution". From physical optics, the far field diffraction pattern of the above field distribution is just its "Fourier transform":

$$
\begin{gathered}
W(x) \supset F\left(f_{x}\right) \propto\left[\operatorname{comb}\left(\frac{f_{x}}{1 / a}\right) \operatorname{sinc}\left(\frac{f_{x}}{1 / b}\right) \operatorname{comb}\left(\frac{f_{x}}{1 / a}\right)\right. \\
\left.\times \operatorname{sinc}\left(\frac{f_{x}}{1 / b}\right) \mathrm{e}^{-\mathrm{i} 2 \pi((a / 2)+d) f_{x}}\right]
\end{gathered}
$$

where $\supset$ stands for "Fourier transform" and $f_{x}$ is the spatial frequency projected on the $x$-axis. The grating's special 


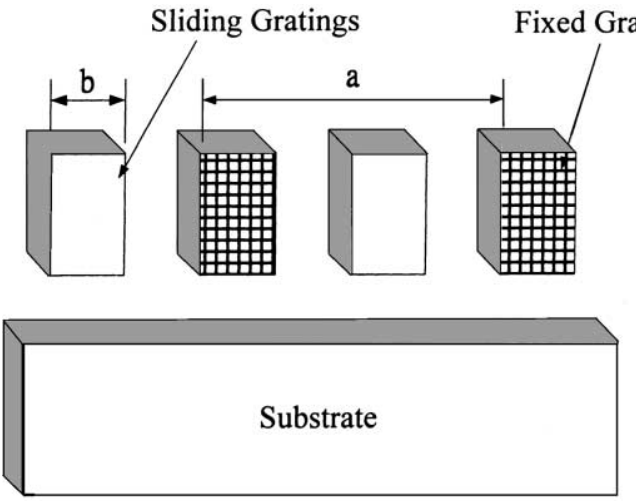

(a)
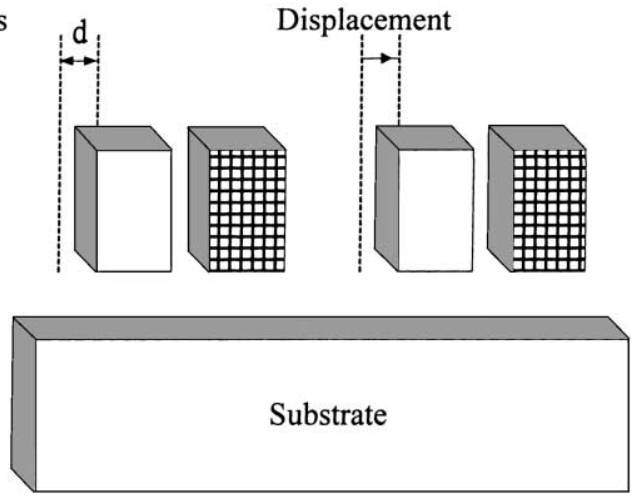

(b)

Fig. 2. The model of the gratings and its physical parameters: (a) the initial position of each gratings; (b) displacement for the movable part.

property of splitting the incident light beam into specific directions is easily understood by considering the characteristics of the comb function. The variation of the diffraction efficiency with the displacement is given by Eq. (6) because parameter $a$ represents the grating pitch and $d$ the displacement between the stationary and sliding gratings. For example, if $f_{x}=1 / a$ and $d=0$ initially, then the phase shift term $2 \pi[(a / 2)+d] f_{x}=\pi$, and the diffraction efficiency is zero. All the odd orders actually disappear when the displacement is zero, corresponding to the grating with double spatial frequency. Furthermore, since the intensity of the diffracted rays is proportional to $\left|F\left(f_{x}\right)\right|^{2}$, substituting in Eq. (6) yields,

$\operatorname{sinc}^{2}\left(\frac{f_{x}}{1 / b}\right)\left\{1+\cos \left[2 \pi\left(\frac{a}{2}+d\right) f_{x}\right]\right\}, \quad f_{x}=\frac{m}{a}$,

where $m$ is an integer.

The diffraction efficiency for every order is proportional to Eq. (7). According to the specific dimensions of the grating in the modulator, substituting $a=12.2 \mu \mathrm{m}, b=2.5 \mu \mathrm{m}$, $d=0$ (initial position, non-shifted) and $d=1.2 \mu \mathrm{m}$ (shifted) into Eq. (7), yields the theoretical diffraction efficiency of the modulator as illustrated in Fig. 3. The derivation shows that $100 \%$ modulation efficiency is achievable for the odd orders. Hence, the micromachined optical

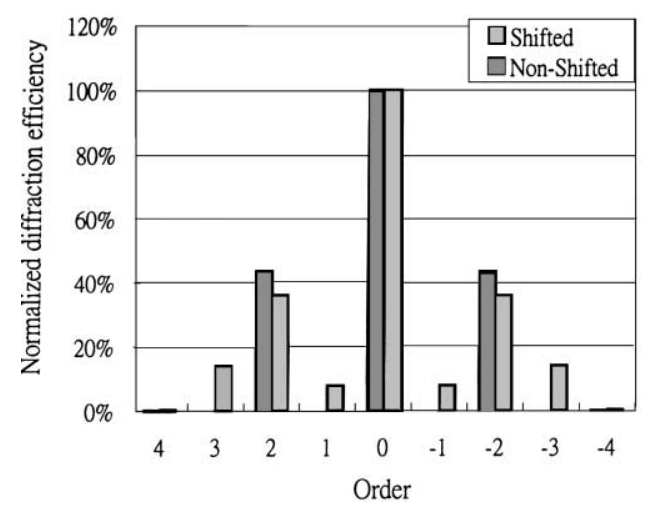

Fig. 3. Theoretical diffraction efficiency. modulator can serve as an optical switch in a direction corresponding to the odd orders.

\section{Fabrication}

The proposed design follows the $0.6 \mu \mathrm{m}$ single poly and triple metals (SPTM) CMOS process. The micromachined optical modulator is developed according to the foundry service and design rules. All post-process procedures require only maskless dry etching and thus avoid the sticking problem.

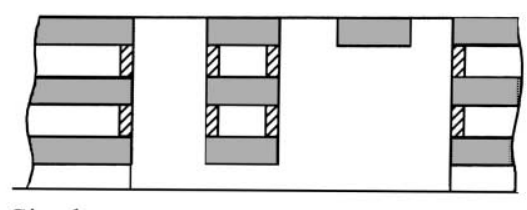

Si substrate

(a)

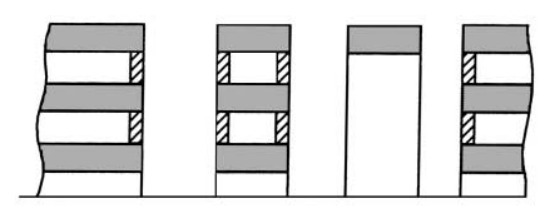

(b)

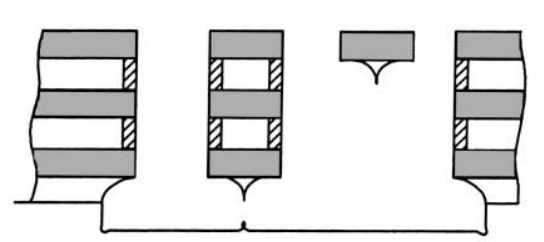

(c)

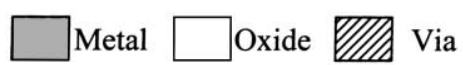

Fig. 4. Schematic process flow: (a) after the CMOS process; (b) anisotropic oxide etching; (c) isotropic silicon etching. 
The micromachined optical modulator is constructed using the $0.6 \mu \mathrm{m}$ SPTM CMOS process available through Taiwan Semiconductor Manufacture Company (TSMC). The process flow in Fig. 4 illustrates development of high-aspect-ratio laminated microstructures in cross section. Fig. 4(a) describes the schematic cross-section view after TSMC process. Two main steps are involved in the postprocess to release the structure. Firstly, all silicon dioxide is etched by anisotropic $\mathrm{CF}_{4} / \mathrm{O}_{2}$ reactive ion etching (RIE) as illustrated in Fig. 4(b), and the structure is defined by the metal layer which acts as a good etching mask for F-based plasma etching. Secondly, silicon substrate is isotropically etched by $\mathrm{SF}_{6}$ RIE to release the whole microstructure as illustrated in Fig. 4(c). Fig. 5 shows a scanning electron micrograph (SEM) of the laminated-suspension microstructures.

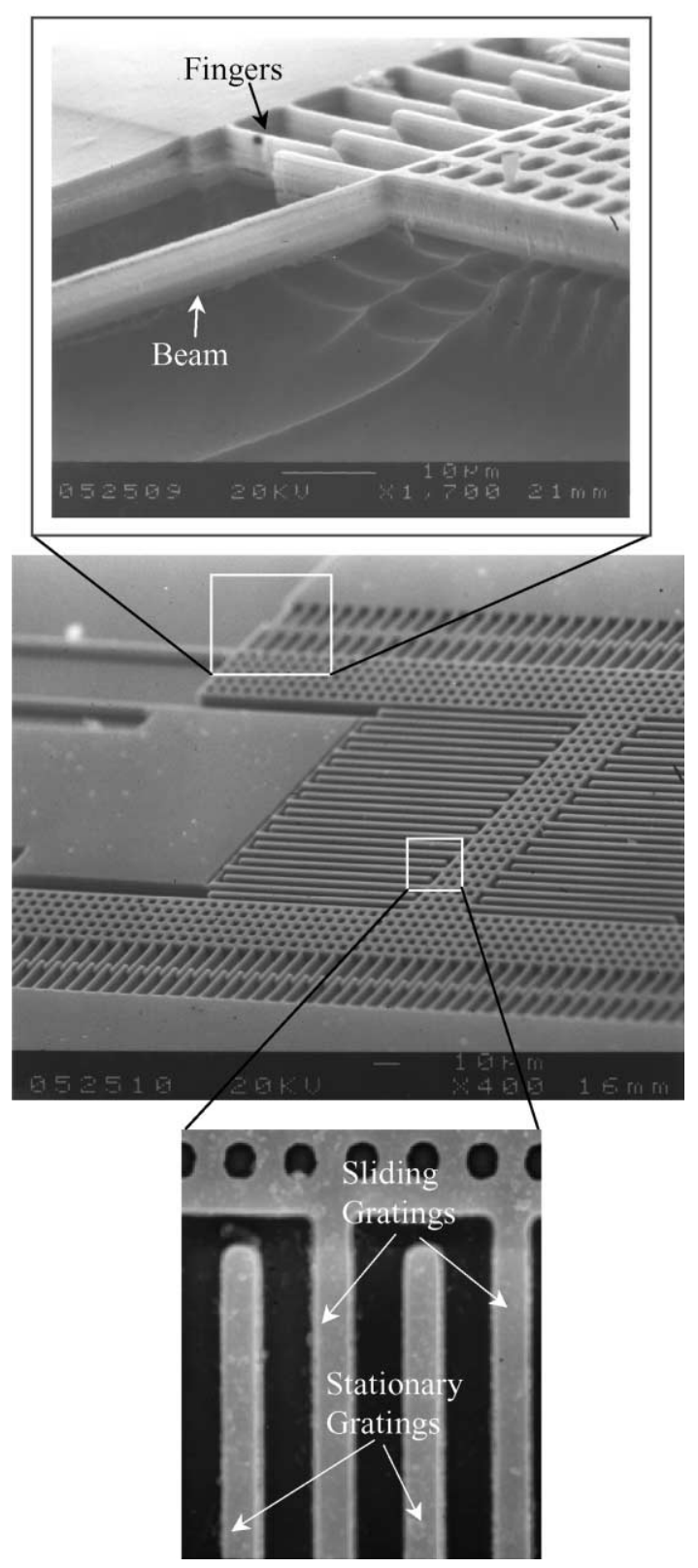

Fig. 5. SEM of the micromachined optical modulator.

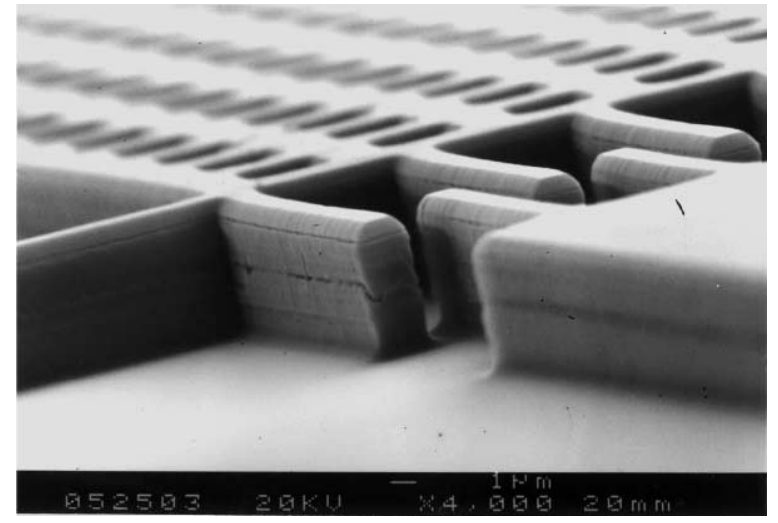

Fig. 6. SEM of laminated high-aspect-ratio microstructure.

\section{Experimental results}

The structure of the micromachined optical modulator enhances the stiffness, which is proportional to the square of the aspect ratio. A 5:1 aspect ratio laminated structure, which theoretically enables a 25:1 flexure stiffness ratio, is shown in Fig. 6. Constructing the optical modulator from the high-aspect-ratio laminated structure can eliminate the gradient deformation caused by a lower $z$-directional stiffness.

The fixed gratings connect to large anchors, in contrast, the free gratings are connected to a small mass with etching holes. The design ensures not only that the movable part is fully released, but also that the fixed part remains attached to the substrate. The bending effect in the micromachined optical modulator could pose a serious problem to the optical field, which requires an accuracy of less than a half-wavelength. Therefore, the gradient deformation and stiffness may limit the working range. Furthermore, metal is deposited at a high temperature, implying the occurrence of residual stress at room temperature. After the sacrificial layer is removed, the residual strains occur in the structure. Modulator with active areas of $100 \mu \mathrm{m} \times 100 \mu \mathrm{m}$ is tested; they exhibit in $z$-directional maximum deformation of about $1 \mu \mathrm{m}$, respectively. This phenomenon causes an additional voltage demand for displacement in the vertical direction (z-direction).

Following the post-CMOS process, a voltage is applied to the actuator of the optical modulator. With electrostatic actuation, the suspended parts slide to the side of the fixed parts to modulate the light. The actuator with $20 \mathrm{~V}$ moves it by $1.2 \mu \mathrm{m}$. Therefore, the comb structure for the actuator is successfully used in this study.

Fig. 7(a) and (b) illustrate the optical measurement devices for the modulator. The measurement involves a trade-off of two factors. First, the area of the grating is very small. Increasing the signal-to-noise ratio requires a light beam in the order of tens of microns in width, reducing the depth of focus and causing an earlier divergence of the diffracted light beam. However, the grating pitch is almost 


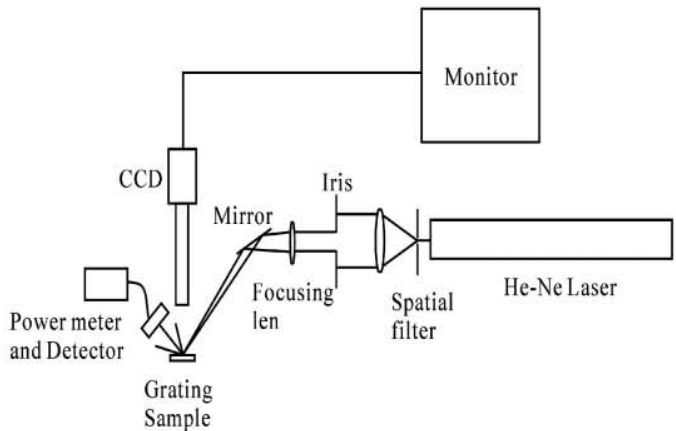

(a)

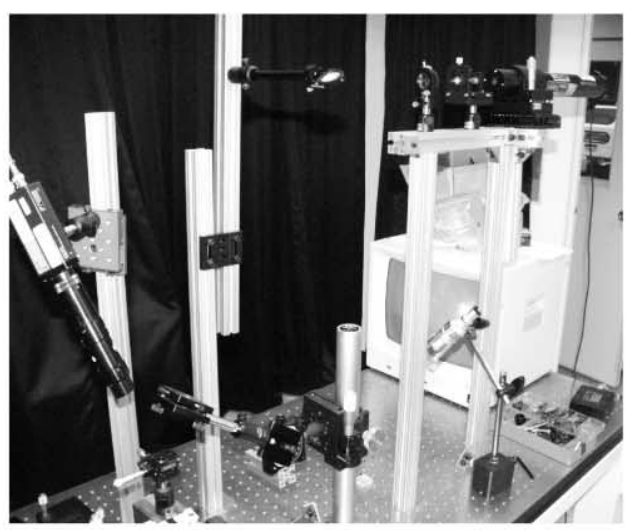

(b)

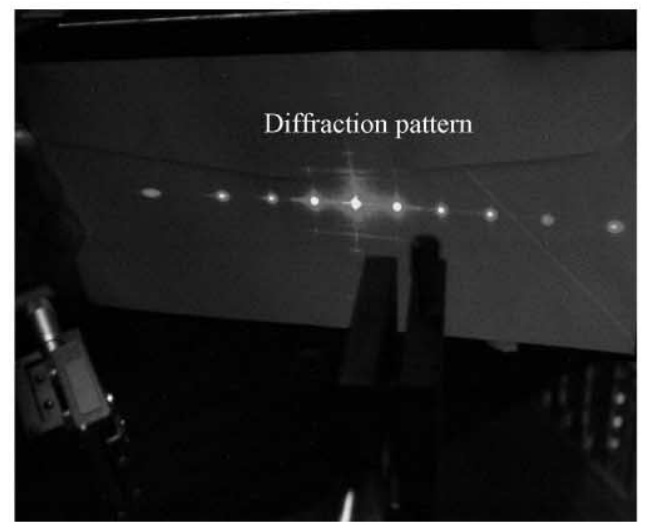

(c)

Fig. 7. (a) Schematic optical measurement devices for the modulator; (b) photograph of the actual optical measurement devices and; (c) diffraction pattern from gratings of the modulator.

10 times the wavelength, so that the difference in the diffracted angle for the neighboring orders is only about several degrees. The most important trick is to control the effective numerical aperture of the focusing lens to prevent the light diverging too seriously. After adjusting controlling the numeric aperture value to optimize the diffraction pattern shown in Fig. 7(c), a power meter can be used to measure the intensity of the separate orders and then compute their diffraction efficiencies.

Fig. 8 presents the experimentally obtained, normalized diffraction efficiency of the pitch-varying grating. The

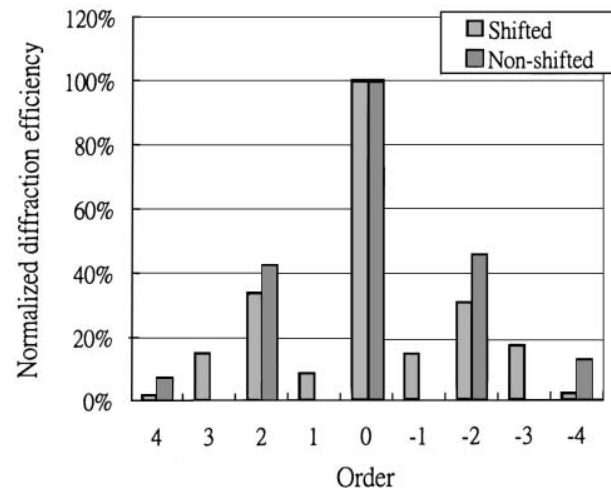

Fig. 8. Experimental diffraction efficiency.

experimental results concerning the fourth and higher orders of the normalized diffraction efficiency apparently deviate from the theoretical values shown in Fig. 3 owing to the residual strains in the modulator after the post-process is completed. However, the experimental results concerning the normalized diffraction efficiency under the fourth order, match closely the theoretical values. The results support the feasibility of the design.

\section{Conclusion}

This study has proposed a micromachined optical modulator with small volume, quick response and fully compatible with the CMOS process. The modulation functions with and without actuation were simulated. Maskless dry etching was the only requirement of the post-process, and only a low voltage of $20 \mathrm{~V}$ was necessary to drive the actuator of the optical modulator. A high-aspect-ratio microstructure was developed to obtain high stiffness in the $z$ direction. Beam widths as small as the minimum allowable metallization line-width given by the CMOS foundry service were generated. The experimental results concerning the normalized diffraction efficiency under the fourth order match closely the theoretical values. The results prove the feasibility of the design.

\section{Acknowledgements}

This work is accomplished with much needed support, and the authors wish to thank the following people: Jennyi Chen, Chiyuan Lee, Hunghsuan Lin, Fuyuan Xiao, Tsungwei Huang, Shihchen Chang and Chunyuan Chi of the Institute of Applied Mechanics, Nation Taiwan University, for their valuable advise and assistance in experiment.

\section{References}

[1] J. Bryzek, Impact of MEMS technology on sociality, Sens. Actuators A 56 (1996) 1-9. 
[2] H.K. Wen, The future of sensor and actuator systems, Sens. Actuators A 56 (1996) 193-197.

[3] B.E.A. Saleh, M.C. Teich, Fundament of Photonics, Wiley, New York, 1991, pp. 815-825.

[4] R.K. Bättig, O. Anthamatten, B. Valk, A reflective modulator based on silicon micromechanics, optical and hybrid access networks, IEE Colloquium 10 (1996) 1-4.

[5] F.S.A. Sandejas, R.B. Apte, W.C. Banyai, D.M. Bloom, Surface microfabrication of deformable grating light valves for high resolution displays, Transducer 93 (1993) 6-7.

[6] D.E. Sene, J.W. Grantham, V.M. Bright, J.H. Comtois, Development and characterization of micro-mechanical gratings for optical modulation, IEEE Trans. Electron Dev. 68 (1996) 222-227.

[7] D.A. Winick, W.M. Teague, P.D. Franzon, A micro-machined approach to optical interconnect, IEEE (1995) 780-785.

[8] D.M. Burns, V.M. Bright, Micromachined thermally based CMOS microsensors, MEMS 97 (1997) 55-60.

[9] J. Bryzek, Development of microelectromechanical variable blaze gratings, Sens. Actuators A 64 (1998) 7-15.

[10] H. Chen, C. Chang, J. Chen, J. Chio, K. Yen, F. Xiao, et al., Fabrication of a micromachined optical modulator using the CMOS process, Transducers 99 (1999) 808-811.

[11] G.K. Fedder, S. Santhanam, M.L. Reed, S.C. Eagle, D.F. Guillou, M.S.-C. Lu, et al., Laminated high-aspect-ratio microstuctures in a conventional CMOS process, Sens. Actuators A 57 (1996) 103-110.

[12] J.W. Goodman, Introduction to Fourier Optics, McGraw-Hill, San Francisco, 1968.

\section{Biographies}

Ching-Liang Dai was born in Chia-Yi, Taiwan, ROC, in 1965. He received the MS degree in the applied mechanics from National Taiwan University, Taiwan, in 1993, and the PhD degree in mechanical engineering from National Taiwan University, in 1997. His PhD dissertation was about the
CMOS microsensors. He is currently an Assistant Professor at the Department of Mechanical Engineering, Oriental Institute of Technology, Taiwan. His research interests are CMOS-MEMS, microsensors and microactuators.

Hung-Lin Chen was born in Nantou, Taiwan, ROC, in 1974. He received the BS degree in mechanical engineering from National Taipei University of Technology, Taiwan, in 1997, and MS degree in applied mechanics from National Taiwan University, Taiwan, in 1999. He joined the Taiwan Semiconductor Manufacture Company, Taiwan, in 2000.

Liang-Bin $Y u$ was born in Taipei, Taiwan, ROC, in 1974. He received the BS degree from the Department of Physics, National Taiwan University, Taiwan, in 1996 . He is currently studying the $\mathrm{PhD}$ program in the Institute of Applied Mechanics, National Taiwan University, Taiwan. His PhD dissertation is about the mechanical and optical properties of subwavelength grating and its applications. His research interests are optomechanical system design and nanostructure analysis.

Chun-Hui Lin was born in Taipei, Taiwan, ROC, in 1976. He received the dual BS degrees both in mechanical engineering and civil engineering from the National Taiwan University, Taiwan, in 1999. Now, he is a graduated student in the Institute of Applied Mechanics of National Taiwan University. His research interests are in the area of optomechanical and optoeletronics system design.

Pei-Zen Chang was born in Chia-Yi, Taiwan, ROC, in 1962. He received the BS degree in civil engineering from National Taiwan University, Taipei, Taiwan, in 1984, and the PhD degree in theoretical and applied mechanics from Cornell University, Ithaca, NY, in 1991. His PhD dissertation was about the mechanics of superconducting magnetic bearings. He joined as a faculty of Institute of Applied Mechanics, National Taiwan University in 1991 and became a Professor in 1999. His current research interests are in the area of micromachined sensors and actuators. 